\title{
A FAMILY WITH THE PROGRESSIVE HYPERTROPHIC POLYNEURITIS OF DEJERINE AND SOTTAS
}

\author{
BY
}

\section{P. D. BEDFORD and F. E. JAMES}

\section{From Cowley Road Hospital, Oxford, and Chippirg Norton, Oxon}

Progressive hypertrophic polyneuritis (Dejerine and Sottas, 1893) is a well established clinicopathological entity which is mentioned in most standard medical textbooks. It is, however, a very rare disease. Since the paper by Russell and Garland (1930) the only cases recorded in British medical journals have been an Australian family (Cooper, 1936). Since Sloane (1939) comprehensively reviewed the literature, communications have appeared sporadically in the world's medical press at the rate of approximately one every three years.

De Bruyn and Stern (1929) have criticized each of the adjectives " progressive", "familial ", " hypertrophic", etc., which have in varying combinations been applied to describe the syndrome of Dejerine and Sottas ; the nature of the polyneuritis has frequently been atypical and has often not conformed with one or more of these descriptions. This communication, however, describes a family in the United Kingdom affected with the disease in its typical form.

The genealogical tree is shown in the Figure. So far as can be ascertained there have been no consanguineous marriages in this family. All its available members have been personally examined. Details have been sought, and information crosschecked concerning all those who have died or for other reasons are not available for examination. The results are also shown in the Figure.

Eight cases of progressive hypertrophic polyneuritis have come to light. The two patients who died long before this enquiry began were probably mildly affected. The case histories of the other six are presented in this paper; two are affected severely, two mildly, and two minimally.

Another hereditary disease-retinitis pigmentosa -also occurs in this family but in only two siblings.

\section{Case Reports}

Case 1 (C.R.H. No. 2459).-Benjamin B. (II.4 in Fig.), a male aged 80 , is an intelligent person who gives a good account of himself. He was perfectly well until the age of 25 when he noticed that his legs occasionally "let me down ". This was the first indi- $\frac{0}{2}$ cation of the weakness of the legs which since then haso been very slowly progressive. At about the same time he began to notice occasional tinglings, numbness, and $\overrightarrow{0}$ "pins and needles" in the hands and feet. Theseparaesthesiae have persisted since then and have always been more obtrusive in the feet than in the hands?

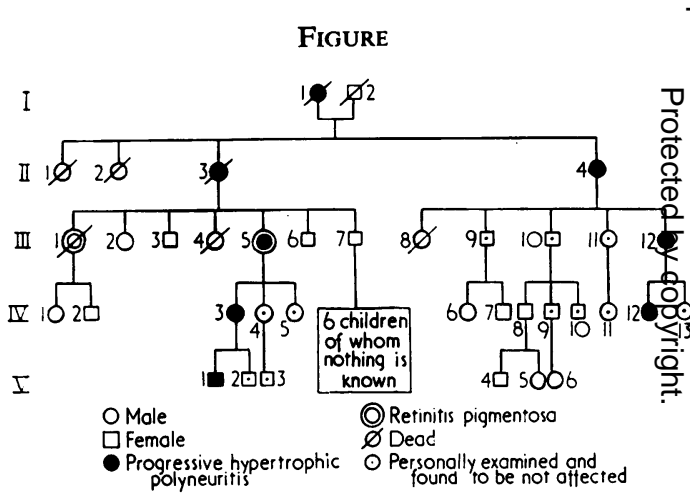

I.1 is stated by II.4 to have had the disease.

I.2 died aged 50-60; she was not affected.

II.1 was killed in an accident when a youth.

II.2 died young and nothing is known about him.

II.3 is stated by II.4 and III.10 to have had the disease.

II.4 is Case 1.

III.1 Very probably had retinitis pigmentosa; he was killed in Worl\$ War 1 aged 25-30.

III. 2 and III.3-nothing is known of these sibs.

III.4 died in infancy aged 6 months.

III.5 is Case 2 and has retinitis pigmentosa.

III.6 and III.7-nothing is known of these sibs. except that III had six children.

III.8 was killed in World War 1 aged about 40 ; nothing else known about him.

III.9, III.10, and III.11 are aged 56, 51, and 47 respectively.

III.12 is Case 5.

IV.1 and IV.2-nothing is known of these sibs.

IV.3 is Case 3.

IV.4 and IV.5 are aged 29 and 30 respectively.

IV.6 and IV.7-nothing is known of these sibs.

IV.8 is aged 28 but nothing else is known of her.

IV.9, IV.10, IV.11, and IV.13 are aged $24,17,8$, and 11 respectivel IV.12 is Case 6.

V.1 is Case 4.

V.2 and V.3 are aged 1 and 9 respectively.

V.4, V.5, and V.6 are aged 5,4 , and 4 respectively; nothing else known of them. 
Some years later there gradually developed an unsteadiness of gait. This ataxia is conspicuous only in the dark or when the eyes are closed; it has never been severe, but is now worse than formerly. There has been no disturbance of sphincter control and no ocular abnormality but his neurological condition has been steadily though extremely slowly progressive. He was in the Royal Air Force in the First World War in Category C3 (he does not know why but " probably because of my feet ").

His account of the family history is as follows-and differs from that given by his nephew (III.5, Case 2). (When challenged with this discrepancy the patient retorted that his nephew "doesn't know what he's talking about" !)

The patient states that his father " used to have difficulty in getting about because of his feet " and that his hands were "all doubled up" but that " this didn't bother him ". When asked whether the deformity of his father's hands and feet was like his own he answers "Yes-but nothing like so bad". His mother was unaffected: he does not know of what she died but she lived to " between 50 and 60 ". So far as is known the founders of the family were not consanguineous; they both however derived from Over Norton, a tiny hamlet near Chipping Norton in Oxfordshire, and the possibility of inbreeding cannot be excluded. His brother (II.3) died " over 80 " and " had feet and hands a bit like mine but nothing like so bad; they didn't bother him-he got used to it". His brother's wife was unaffected; she " died young-about 50 ". As far as he is aware, all his children and grandchildren are normal, but he believes that his nephew (III.5) has feet and hands "a bit like mine-but not so bad". His account is corroborated by III.10 (his daughter-an unaffected female) who states that " Uncle Jim, i.e., II.3, used to walk like father and throw his feet about".

On examination the cranial nerves reveal no abnormality and, in particular, the pupils and ocular fundi are normal and there is no nystagmus.

There is symmetrical weakness and wasting of distal distribution in the arms and legs, the legs being more severely affected than the arms. The hands are more affected than the forearms, and the upper arms and shoulders not at all. There is bilateral flaccid foot drop with conspicuous wasting of the muscles below the knee and only slight weakness and wasting of both quadriceps femoris. The muscles of the hips and trunk are normal.

There is slight impairment of sensibility to pin-prick in the fingers of both hands and considerable impairment in both legs below the knee. (The proximal limit of hypo-algesia is ill-defined in both arms and legs.) Touch, deep pain, and temperature sensations are reasonably well perceived. Vibration perception is impaired in both hands and absent in both legs below the knee. Postural sensibility is normal in the hands but is grossly impaired in the toes, ankles, and knees.

There is complete tendon areflexia except for a sluggish jaw jerk and a barely perceptible right triceps jerk. The cutaneous reflexes are preserved; the plantar responses are flexor. Romberg's sign is positive.
His gait is high stepping and rather wide based; it becomes grossly ataxic when the eyes are closed. There is incoordination of "sensory type" in that the fingernose and heel-knee tests are poorly and inaccurately performed with the eyes closed, but are accurately carried out under visual control. There is no evidence of cerebellar disturbance. The external popliteal nerves, as they wind round the fibulae, and the ulnar nerves at the elbow are grossly hypertrophied. The calves are not tender. There is a single telangiectasis of the forehead but no abnormality of any other system. The cerebrospinal fluid is normal and the Wassermann and Kahn reactions negative.

A biopsy specimen of the cutaneous division of the musculo-cutaneous nerve of the left foot is reported on as follows (S.H. No. 1334) :-

"A length of peripheral nerve $2 \mathrm{~cm}$. long by $3 \mathrm{~mm}$. wide by $1 \mathrm{~mm}$. thick. It is made up of several well separated bundles. No nodularity is visible macroscopically. Histology shows the characteristic onion formation of hypertrophic neuritis. There is some degeneration with an increase in the number of Schwann nuclei and also some increase in the collagen around the fibres."

This is a severe symmetrical polyneuropathy of distal distribution affecting motor function more than sensory and the legs more than the arms. The condition has been very slowly progressive. The peripheral nerves are grossly hypertrophied. Biopsy confirms the diagnosis of progressive hypertrophic polyneuritis.

Case 2.-Albert B. (III.5 in Fig.), a male aged 58, is the nephew of Case 1 .

His infancy was normal except that he walked rather late. In childhood he noticed that he stumbled at games because " my feet dropped ". He was never as nimble, fast, or agile as other children. In adolescence he began suffering from "pins and needles", numbness and tingling in the legs, which symptoms have continued ever since but are less obtrusive now than formerly. There have been no paraesthesiae in the arms. At the same time, and of gradual onset, he noticed himself to be unsteady on his feet. This ataxia is of "sensory" type in that he is perfectly well able to preserve his balance during the day or when his eyes are open, but cannot do so in the dark or with his eyes closed. Weakness of both legs together with bilateral foot drop developed very gradually over the years but became much worse at about the age of $\mathbf{4 0}$ to 50 following a period of immobilization for a Pott's fracture of the left ankle. His disability has been steadily but very slowly progressive and is even now not severe. There has been no disturbance of sphincter control. He joined the Army in the First World War in medical category A1 but was demobilized in Category B2 because of a nonpenetrating shrapnel wound in the posterior part of the head. He first noticed a defect in night vision some years ago and this has become slowly but steadily worse. At the same time he noticed difficulty in rapid visual accommodation for near objects. This is due to a congenital pigmentary defect of the retina (retinitis 
pigmentosa) for which he has attended the Oxford Eye Hospital.

His account of the family history differs from that given by his uncle (II.4, Case 1) and his cousin (III.10). He does not remember his grandfather very well but he believes that he had nothing wrong with his hands or feet; he cannot remember his grandmother. He states that his father " had abnormal thumbs" but walked well, could pick up nails to mend his own boots, and could climb ladders to clean lamps until he was 74 years of age : he died aged 84 . His mother died of heart failure when aged 50. His eldest brother, who was killed in the First World War, " had eyes just like mine ; he couldn't see in the dark either", but other than this visual defect " he was perfectly sound". Initially he stated confidently that except for his uncle no one else in the family was affected. At a later date, however, when confronted with other affected members of the family, he agrees that his account is incomplete and probably erroneous, and offers the shrewd observation that "you can see it in the thumbs first".

On examination he strongly resembles his uncle. $\mathrm{He}$ is a heavily built, powerful, intelligent man of somewhat euphoric disposition. He has a single telangiectasis on the forehead and the scar of an old shrapnel wound posterior to the inion. There is no abnormality of any system other than the neurological.

His night vision is poor but in full light his vision is excellent except for near objects. There is a fully correctible myopia of about three dioptres for which he wears " reading glasses". There is slow, defective, and incomplete ocular accommodation for near objects. The ocular fundi show the classical pigmentary changes of retinitis pigmentosa; there is no optic atrophy. Otherwise the cranial nerves are normal; the pupils react normally to light and there is no nystagmus.

There is wasting of the thenar eminences of both hands and wasting of the legs below the knees with bilateral flaccid foot drop. There is moderate diffuse weakness of the muscles of the hands and forearms and considerable weakness of the legs and feet below the knees. The power of the rest of the musculature is excellent.

The painful quality of pin-prick is not perceived over both hands and the medial borders of both forearms, and over the feet and medial aspects of both legs below the knees ; the upper limits of this hypo-algesia are not clearly defined. Light touch is impaired over the same areas but temperature sensibility is preserved. Vibration is less well perceived in the hands than proximally and is absent in both legs below the knees. Postural sensibility is normal in the arms but is impaired in the legs ; he is unable to point accurately to his great toes with his eyes closed.

There is complete areflexia of the tendon jerks.

The cutaneous reflexes are preserved and the plantar responses flexor. Coordination is impaired in the arms and legs, judged by the finger-nose and heel-knee tests, only when his eyes are closed; the tests are accurately performed with the eyes open.
Romberg's sign is positive.

There is no evidence of cerebellar disturbance.

His gait is wide based and high stepping and is grosslyes ataxic with his eyes closed.

The ulnar nerves and to a greater degree the external popliteal nerves as they wind round the neck of thes fibulae are grossly enlarged.

This is a severe mixed sensory and motor polyneuro pathy of very gradual progression associated with con spicuous hypertrophy of the peripheral nerves.

The patient also has retinitis pigmentosa.

Case 3.-Ronald Albert B. (IV.3 in Fig.), a malę aged 34, is the son of Case 2.

This patient was born with "funny feet". He has never been able to run properly and this disability is most marked when he tries to run fast. His hands have " always been clumsy" but " are no worse now thar" they always were". This clumsiness applies only " with little things like a pin ; I don't usually get it first time ". He also has some difficulty with small buttons, e.g., thosఱ్ on a shirt. He feels that his symptoms are trivial and. have been quite stationary throughout his life. H马 denies paraesthesiae. He is not unsteady in the dark? His vision is excellent both by night and by day; hiso colour vision is normal. He plays games but "I am little clumsy on my feet" (he has someone to run fo him when batting at cricket but " manages fairly we when he fields in the slips). He served for six yearsin the late war in an anti-aircraft battery in the Army,ain medical Category B7 because of his feet. He does aD full day's work as a heavy manual labourer, using his arms and shoulders rather than his feet. His work응 so heavy that he occasionally suffers salt depletion cramps. He is inordinately proud of his physical proves and appears to have somewhat over-compensated for the disability of his feet.

On examination he is a slightly built, intelligent map who conceals an anxiety about his physical condition under an aggressive euphoria. There is a single " spide⿳亠二口 angioma " on the right cheek but no abnormality else where than in the nervous system. The cranial nerve $\$$ are normal ; in particular the ocular fundi and pupils are normal and there is no nystagmus. There is diffuse wasting of the small muscles of both hands (more conspicuous in the right than the left), and the opponens pollicis muscles are particularly affected. The palmar. surfaces of the hands are flattened and the dorsal aspects. show marked hollowing between the extensor tendons? His feet are clawed with callosities on both soles, and there is bilateral flaccid foot drop-again more marke $\Phi$. on the right than the left. The small muscles of both feet and of the anterior tibial group of muscles in both legs are wasted. There is slight weakness of extension and even less of flexion of the hands, wrists, and fingers? and moderate weakness of dorsiflexion of both feet $\supset$ Otherwise power is excellent throughout.

The painful quality of pin-prick is poorly perceive $\overline{\bar{J}}$ over the distal phalanges of the hands and in both leg§ below the knees-particularly in the feet. Touch and temperature sensibilities appear to be unimpaired心 
Postural sensibility is normal in the arms but is defective in the legs; he fails to point accurately to his great toes with his eyes closed. Vibration perception is impaired in both feet. There is complete areflexia of the tendon jerks but the cutaneous reflexes are preserved. The plantar reflexes are flexor.

When the eyes are closed coordination is impaired (finger-nose test; heel-knee test), but the tests are accurately performed with the eyes open.

His gait shows the high stepping quality of mild flaccid foot drop and is ataxic when the eyes are closed but not when they are open.

Romberg's sign is not present.

There is no evidence of cerebellar disturbance.

The ulnar nerves at the elbow and the external popliteal nerves as they wind round the fibulae are grossly hypertrophied (the right ulnar nerve is approximately $\frac{3}{8}$ in. (nearly $1 \mathrm{~cm}$.) in diameter).

This is a mild mixed sensory and motor polyneuropathy present since childhood and showing little or no progression. It is associated with gross hypertrophy of the peripheral nerves.

Case 4.-Barbara B. (V.1 in Fig.), a female aged 10, is the daughter of Case 3 .

Her mother states that she " has feet like her father's" and that she is unable to run as fast as other children ; this was first noticed when she began to walk-which she did rather late-at the age of 5 . She is otherwise perfectly well.

On examination she is a pleasant, well built, intelligent child. She is left handed. The only abnormalities detected are slight weakness of dorsiflexion of both feet ; complete areflexia of the tendon jerks with preservation of the cutaneous reflexes; slight ataxia when walking with the eyes closed, with a slight tendency to flaccid foot drop; some impairment of vibration perception in the feet ; slight impairment of large joint postural sensibility (as manifested by failure to point accurately to the great toes with the eyes closed) ; slight " sensory" ataxia as shown by the heel-knee test with the eyes closed ; and conspicuous enlargement of the left external popliteal nerve at the neck of the fibula.

There are no other symptoms ; there are no paraesthesiae. No other abnormality can be detected and in particular there is no obvious muscle wasting. The pupils and ocular fundi are normal and there is no nystagmus.

This is a minimally affected case of progressive hypertrophic polyneuritis, but whether or not it will subsequently progress is uncertain.

Case 5.-Dennis B. (III.12 in Fig.), a male aged 40, is the son of Case 1 .

$\mathrm{He}$ is an engineer's labourer who is aggressively confident that "there's nothing wrong with me". His feet have been "slightly deformed" for as long as he can remember but have given him no trouble whatever. His infancy and childhood were uneventful. His athletic ability at school was average. He joined the Army in the late war in medical Category $\mathrm{Al}$ but was regraded B7 because of his feet.
On direct questioning he admits to paraesthesiae in the left leg and both hands "for some years". This is his only symptom ; it comprises tingling, "pins and needles", and numbness unrelated to posture, sleep, or activity. The paraesthesiae have always been mild but are slightly more obtrusive now than formerly. $\mathrm{He}$ is otherwise perfectly well.

On examination he is of small stature (64 in.) but is well proportioned. He bears a strong facial resemblance to his father. There is no abnormality in any system other than the neurological.

The cranial nerves are normal as in particular are the ocular fundi and pupils, and there is no nystagmus. There is flattening of the thenar eminences of both hands with conspicuous weakness and wasting of the opponens pollicis and flexor brevis pollicis of both hands. There is moderate diffuse weakness of both flexion and extension of the fingers of both hands (and more especially of flexion), but no muscular wasting in the upper limbs can be detected elsewhere. There is bilateral pes cavus with slight flaccid weakness, and wasting of the dorsiflexors of both feet. There is slight sensory impairment in the hands in that pain (pin-prick) and light touch are poorly perceived in the first and second fingers of both hands. Vibration perception and postural sensibility are slightly impaired in the legs below the knees, but not elsewhere, and all other sensory modalities appear to be normal.

All the tendon reflexes are extremely sluggish even on reinforcement. The cutaneous reflexes are preserved and the plantar responses flexor. Coordination is normal in the arms but is slightly impaired in both legs. This mild ataxia is of "sensory" type in that the tests are accurately performed with the eyes open but not with the eyes closed.

Romberg's sign is not present.

There is a suggestion of foot drop on walking, but otherwise the gait is normal. The right external popliteal nerve, as it winds round the neck of the fibula, and the cutaneous division of the left musculo-cutaneous nerve of the foot are grossly enlarged; the other peripheral nerves appear to be normal.

This i a long-standing, mild, mixed polyneuropathy affecting the hands and feet, associated with conspicuous hypertrophy of two peripheral nerves.

Case 6.--Robert B. (IV.12 in Fig.), a male aged 14, is the son of Case 5 .

This boy admits to no symptoms. $\mathrm{He}$ is a member of the school association football team and passed his infant " milestones" without incident and at the normal age.

On examination he is an intelligent, alert youth of good physique. The only abnormalities detected are slight flattening of the thenar eminences with weakness and wasting of the muscles of both thumbs (especially of the flexor brevis and the opponens pollicis), moderate enlargement of both median nerves at the wrist, and impairment of vibration perception in the feet. All the tendon reflexes are sluggish, even on reinforcement. The cutaneous reflexes are normal and the plantar 
responses flexor. The other peripheral nerves do not appear to be enlarged; the ocular fundi and pupils are normal and there is no nystagmus. His feet are normal and there is no foot drop.

This is a minimally affected case of progressive hypertrophic polyneuritis, but whether or not it will subsequently progress is uncertain.

In none of the cases was fasciculation of muscles visible. There were no neurofibromata, no abnormal cutaneous pigmentation, and no spinal deformity.

\section{Discussion}

There are many inconstant features of the syndrome of Dejerine and Sottas as is indicated by the many adjectives used to describe the polyneuritis. The only constant clinical feature is muscular weakness and wasting which may be slight, moderate, or severe. Sensory symptoms and signs are usually present but rarely obtrusive and frequently absent. Thickening and enlargement of one or several of the peripheral nerves frequently occurs and may be extreme. When present this feature is virtually pathognomonic and clearly distinguishes the syndrome from Tooth's (1886) “ peroneal muscular atrophy ". But enlargement of the peripheral nerves may not be clinically apparent if of minor degree, and indeed may not occur at all. The histological picture, however, is characteristic and pathognomonic although biopsy may be inconclusive as not all the nerves may be affected at any one time nor to the same extent. De Bruyn and Stern (1929) criticize the term "interstitial neuritis" since the lesion is essentially a hypertrophy of the sheath of Schwann. For this reason Bielschowsky (1922) considers that the disease is related to neurofibromatosis. The adjective "progressive" as applied to the disorder is also open to criticism, for although there is usually a gradual and very slow deterioration, the progress of the disease may halt spontaneously at any stage, and may thereafter show no advance whatever.

Although usually a heredo-familial disorder, many of the recorded cases have occurred sporadically and in isolation in fully documented and otherwise unblemished family trees. Thus the adjective " familial" is also open to criticism. From a study of the published families the mode of inheritance would appear to be variable. Snyder (1941) states that it is usually a recessive characteristic ; and in view of the many isolated cases published this may well be so. In many families, however, the mode of inheritance would appear to be dominant if the definition be accepted that a characteristic is dominant if it affects parent and child or several sibships in the same generation.
In the family described here the disease appear to be inherited as a heterozygous dominant charac teristic if the statements of II.4 (Case 1) and III.18 be accepted as true. The initial discordant accoun of the family history given by III.5 (Case 2) intro duces the possible anomaly of II.3 being unaffectedf The subsequent purity of heterozygous dominanos in the line of II.3 would be difficult to explain excep on the basis of an adulterous relationship between his wife and an affected male, e.g., II.4 (Case F: which is of course a reasonable alternative hypof thesis. Further information regarding this posst: bility may become available from a study of the blood groups of the members of the family which is at present being carried out. It is, howeve? highly probable that II.3 was in fact affected, fơ both II.4 and III.10 have independently asserted this while III.5 originally denied it but subsequently retracted his denial. The chances of any chilad being affected in this family are difficult to forecasi accurately. Although no child can have the disease unless the father or the mother is affected, the likelihood of its reappearance in the lines of IV.9 and III.12 seems considerable.

It is of interest to note that after the disco perer of Cases 1 and 2 (II.4 and III.5) the initial enqüiry and admittedly superficial examination of the odher members of the family conducted by one of authors failed to disclose the remaining cases. कुh led to the erroneous genetic explanation of the ofur rence by spontaneous mutation of a recessive of low penetrance. Only after very careful furthe enquiry and examination did the true facts come 8 light and the heterozygous dominant transmissiof be revealed. By analogy, it is possible that sonere of the sporadic and isolated cases which have been published and for which a recessive mode of inher:ance has been postulated (Snyder, 1941) were th result of the same error. It cannot be too strongf emphasized that in an investigation of a hered $\bar{\sigma}$ familial disorder the histories must be cross-checked at every possible point and that careful persona examination of all the available members, whether alleged to be unaffected or not, is an essential requisite.

Several cases of the syndrome of Dejerine and Sottas have been described in which pupillagy anomalies have been noted, such as anisocoria, and very slow reaction to light and convergence (Francois and Descamps, 1949). At one time the Argyll-Robertson pupil was erroneously thought be an integral part of the disorder (De Bruyn and Stern, 1929). No case however has previously beep reported with retinitis pigmentosa as had Case ${ }^{2}$ (III.5) whose eldest brother (III.1) very probably 
was similarly affected. Retinitis pigmentosa is carried by a recessive gene, and the association in this family of the two hereditary conditions-the heterozygous dominant polyneuritis and the recessive retinitis-is probably fortuitous.

\section{Summary}

A family of five generations is described in which progressive hypertrophic polyneuritis (syndrome of Dejerine and Sottas) is inherited as a heterozygous dominant characteristic.

Eight members of the family were affected, two severely, four mildly, and two young children minimally. Case histories are presented of the six who were available for examination. Histological confirmation of the diagnosis was sought and obtained in one case.
One affected male also has retinitis pigmentosa and it is highly probable that an otherwise healthy brother had the same inherited retinal disorder.

We are grateful to Dr. P. M. Daniel for the pathological report, to Dr. W. Ritchie Russell for his helpful criticism, and to Dr. F. Whitehead and Mr. E. A. Bevan for their opinion on the genetics of this family.

\section{REFERENCES}

Bielschowsky, M. (1922). J. Psychol. Neurol. ( $L p z$ ), 29, 182.

Cooper, E. L. (1936). Brit. med. J., 1, 793.

De Bruyn, R. S., and Stern, R. O. (1929). Brain, 52, 84

Dejerine, J., and Sottas, J. (1893). C.R. Soc. Biol. (Paris), 9 ser., $5,63$. François, J., and Descamps, L. (1949). Acta neurol. psychiat. belg., $49,648$.

Russell, W. R., and Garland, H. G. (1930). Brain, 53, 376.

Sloane, P. (1939). J. nerv. ment. Dis., 90, 429.

Snyder, L. H. (1941). Medical Genetics. Duke University Press, Durham, North Carolina.

Tooth, H. H. (1886). The Peroneal Type of Progressive Muscular Atrophy. Lewis, London. 